\title{
Ship to Ship Maritime Communication for e-Navigation Using WiMAX
}

\author{
Myeong Soo Choi, Sun Park, Yeonwoo Lee and Seong Ro Lee \\ Mokpo National University, South Korea \\ \{mschoi, sunpark, ylee, srlee\} @mokpo.ac.kr
}

\begin{abstract}
Maritime wireless communication is different from terrestrial wireless communication, due to difference of environments. Nevertheless technologies based on WiMAX) IEEE 802.16j) for ship to ship maritime communication promised to deliver high data xates oyer large distances and deliver multimedia services and are expected to use'e-Navigation system. This paper focuses to simulate on IEEE $802.16 \mathrm{j}$ for ship to shiR maritime communication under various sea states. We describe BER level according to sea states under Rician fading channel model. $B E R$ patterns are designed using MATLAB.
\end{abstract}

Keywords: IEEE 802.16j, e-Navigation,Maritime Cemmunication, Ship to Ship, WiMAX

\section{Introduction}

Recently, the maritime communication between ship to ship/shore is technically limited to a high cost. In this respect various research about how to reduce a high cost is in progress. To solve this problem, we adopt WiMAX (LEEE 802.16j) over the maritime environment. The IEEE $802.16[1,2]$ aims to provide d high transmission rate and wide coverage for wireless communications.

Current maritime wireless communication systems are based on MF, HF, VHF radios for ship to shore communications offshore, and satellite communications for long range ship to ship and ship to shore communications. These maritime radio technologies cannot support the e-Navigation service due to the lack of bandwidth. And using satellite is too expensive for commercial services To solve these problems, improved maritime communication technology is néeded to transmit large multimedia data.

We present /WiMAX (IEEE 802.16j) technology and existing maritime wireless communication technology in Section 2. In Section 3, we introduce system description for our simuation. In Section 4, we present simulation results using MATLAB. Finally in Section 5, we conclude this paper.

\section{WiMAX and Maritime Wireless Communications}

\subsection{WiMAX (IEEE 802.16j)}

The relevant standards include IEEE 802.16d, 802.16e, 802.16j, etc. The 802.16d targets at the fixed wireless access while the 802.16e counterpart is designed to support the mobile applications. It is known that the single hop communication is often of limited geographical size. In this respect, a relay approach by introducing the low-cost relay station (RS) into the 802.16e network to increase range and enhance transmission capacity between a base station (BS) and mobile station (MS) in a cost-effective manner is highly desirable[3]. Such RS- 
enabled multi-hop networks have been the focus of the recently established IEEE $802.16 \mathrm{j}$ standard working group [4-6]. Specifically, this standard is based on the legacy 802.16e but extends it to support the mobile multi-hop relay (MMR) operation. Due to its high transmission rate, wide coverage, mobility and flexible deployment, the 802.16j MMR network are regarded as a promising alternative for broadband wireless access in a wide area, especially in a maritime environment. An IEEE $802.16 \mathrm{j}$ network includes three types of stations, i.e., BS, RS and MS. The MS is also called as the end user station, while all the other stations are called as the RSs. The station to which an RS transmits in the uplink is its superordinate station while transmits in the downlink is its subordinate station. In such a mobile multi-hop transmission network, the RS plays a critical role in forwarding traffic between BS and MS[3].

\subsection{Maritime Wireless Communications}

Conventional maritime wireless communication is based on voice communication, which is using radio devices of MF, HF, VHF and satellite system. The radio devices loaded to a vessel are decided according to the size and sailing area of vessels. IMO and ITU defined these radio types for sailing vessels[7].

Table 1. IMO definition of sea ârea[7]

\begin{tabular}{ccc}
\hline Sea Area & Technology \\
A1 & Coast $(50 \sim 100 \mathrm{Km})$ \\
A3 & Offshore $(200 \sim 400 \mathrm{Km})$ \\
North Latitude $70^{\circ}$-South Latitude $70^{\circ}$ \\
Worldwide
\end{tabular}

IMO defined the maritime pireless communication according to technology and communication coverage as in table I. A1 and A2 are massive complex areas of each country where small ships are sailing. $A 3$ and $A 4$ areas are ocean where large ships are sailing [7].

TRITON [8] implemented mesh network for maritime communication using a ship, lighthouse, buoys as conmunication nodes based on IEEE 802.16 and IEEE 802.16e. Current satellite systems such as the INMARSAT GAN system provides only up to $64 \mathrm{kbps}$ of data bandwidth per/data link. And that bandwidth is shared among the nodes, so the real bandwidth becomés lower [8].

ESA (European Space Agency) [9] introduced Wired Ocean Project. They suggest low costand broadband IP based TV, internet and communication services using hybrid satellite and DVB (Digital Video Broadcast) systems in the ocean. But the cost is still expensive due to Wired Ocean needed TVRO (TV Receive Only) antenna for implementation.

\section{System Description}

In MIMO systems, a transmitter sends multiple streams by multiple transmit antennas. The transmit streams go through a matrix channel which consists of all $n_{t} n_{r}$ paths between the $n_{t}$ transmit antennas at the transmitter and $n_{r}$ receive antennas at the receiver. Then, the receiver gets the received signal vectors by the multiple receive antennas and decodes the received signal vectors into the original information [10]. 
The MIMO system model can be described as:

$$
\mathrm{r}=\mathrm{sH}+\mathrm{n}
$$

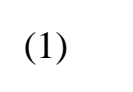

where $r$ is $n^{\text {th }}$ received signal vector, sis $n^{\text {th }}$ transmitted vector, $H$ is $n_{t} * n_{r}$ channel matrix and $\mathrm{n}$ is the Gaussian noise vector.

The relation between the trantsmitter and receiver is given by:

$$
\begin{aligned}
& \mathrm{r}_{3}=\mathrm{s}_{1} \mathrm{~h}_{11}+\mathrm{s}_{2} \mathrm{~h}_{12}+\ldots+\mathrm{s}_{\mathrm{n}} \mathrm{h}_{\mathrm{n}} \\
& \mathrm{r}_{2}=\mathrm{s}_{1} \mathrm{~h}_{21}+\mathrm{s}_{2} \mathrm{~h}_{22}+\ldots+\mathrm{s}_{\mathrm{n}} \mathrm{h}_{\mathrm{n}}
\end{aligned}
$$

The channel matrix $H^{\prime}$ can be expressed as $n *$ nr, with nt inputs and nr outputs. The transmitted data can be fully recovered by expressing the channel in matrix form. Thus the channel matrix can be represented as:

$$
H=\left[\begin{array}{cccc}
h_{11} & h_{12} & \cdots & h_{1 m} \\
h_{21} & h_{22} & \cdots & h_{2 m} \\
\vdots & \vdots & \ddots & \vdots \\
h_{n 1} & h_{n 2} & \cdots & h_{n m}
\end{array}\right]
$$

Where $h_{h m}$ is the attenuation and phase shift between the $n^{\text {th }}$ transmit and $m^{\text {th }}$ receive antenna.

The WiMAX multiple antenna technique is often categorized as either open loop or closed loop. Most common open loop MIMO techniques include STC (Space Time Coding) and Spatial Multiplexing. Considering open loop system, the WiMAX standard includes two versions of open loop MIMO techniques known as Matrix A and Matrix B, referring to STBC technique and SM (Spatial Multiplexing) MIMO technique respectively.

In MIMO Matrix A, the data stream is replicated and transmitted over multiple antennas. Mathematical algorithms are used to encode each redundant data streams known as STBC (Space Time Block Codes). This multiple transmission of the coded data streams, raises the opportunity for the receiver to identify the signal. 
In MIMO Matrix B, the signal to be transmitted is split into multiple data streams, where each data stream is transmitted from a different base station transmit antenna.

In a maritime wireless communication environment, the channel state or quality over a communication link is affected by three attenuation factors, i.e., path loss, path shadowing and multipath fading [1, 11]. Path loss is due to the propagation distance between the transmitter and receiver station although the communication link between them is in the line of sight (LOS) of the transmitter. Path shadowing is caused by the large obstacles between the transmitter and receiver station and may cause more attenuation than path loss. Multipath fading is caused by the multiple receptions of the original signal due to the sea surface reflection[3].

Two stations A and B are placed over the sea surface. Let A denote a transmitter station and $B$ represent a receiver station. According to the typical sine wave movement patern [11] of the sea surface, each station goes up and down along sea surface in a peribdical manner.

To determine the signal propagation manner between station $A$ and $\mathrm{B}$, we should first determine their positions. As for the coordinate system used in Fig. 2 , point $\mathrm{O}(0,0)$ denotes the selected coordinate origin. $\mathrm{X}_{\mathrm{i}}(\mathrm{t})$ and $\mathrm{Y}_{\mathrm{i}}(\mathrm{t})(\mathrm{i}=\mathrm{A}$ or $\mathrm{B})$ denote the horizontal and vertical coordinate of the station respectively. The vertical coordinate $\mathrm{Y}_{\mathrm{B}}(\mathrm{t})$ of station $\mathrm{B}$ is determined according to the sea surface wave movement as

$$
\mathrm{Y}_{\mathrm{B}}(\mathrm{t})=\mathrm{a} \sin \left[2 \pi * \bmod \left(\mathrm{X}_{\mathrm{B}}(\mathrm{t}),(\mathrm{\lambda}) / \mathrm{\lambda}+2 \pi * \operatorname{mog}(\mathrm{T}, \mathrm{T}) / \mathrm{T}\right]\right.
$$

Where parameters a, $\lambda$ and $\mathrm{T}$ denote the wave amplitude, wave length and wave cycle of the sea surface movement[3].

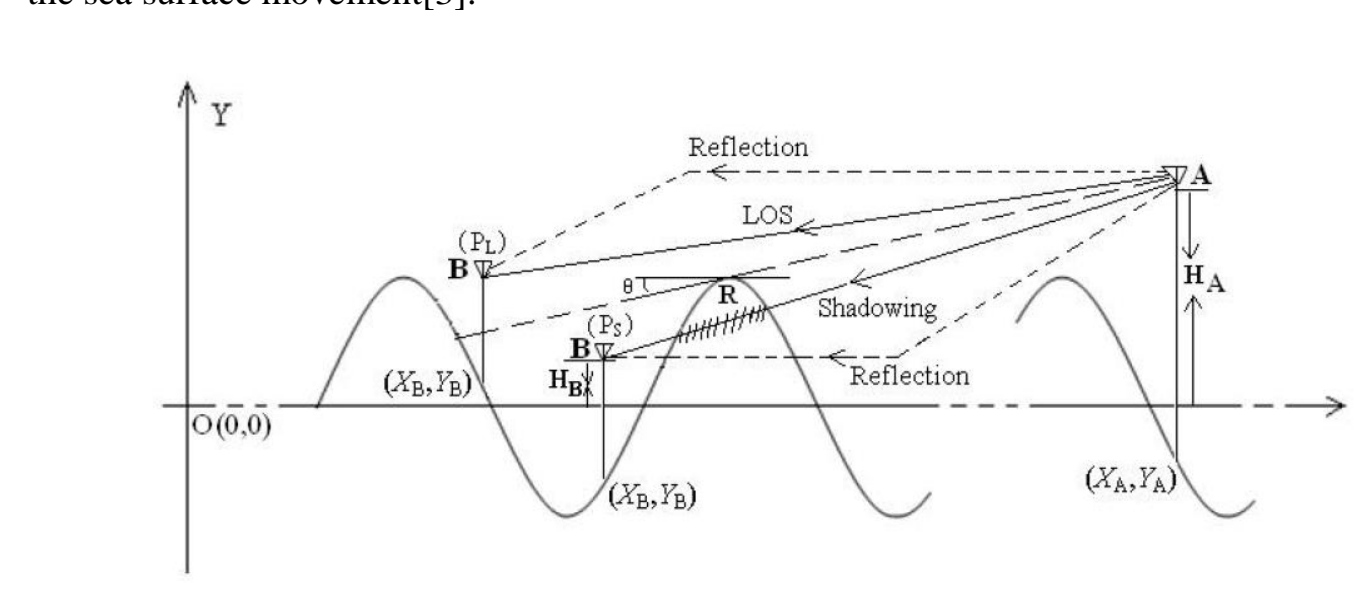

\section{Figure 2. Three types of signal propagation manners, i.e., LOS, shadowing and reflection propagation [3]}

Ocean waves are dependent upon the wind. These ocean waves relate to the motion of a ship in the sea. Ships tend to have some form of rocking motion, which affects the communication link between the ship and the shore. The ocean wave is often depicted as a typical sine wave movement pattern; however, by experiments the wave shape is described as a Trochoid, whose properties are known to be similar to that of sea waves. The roughness of the sea surface are measured by so called sea states, which is characterized by parameters of significant sea wave height, average sea wave length, and average sea wave period. In this paper, since we do not have any real data, we use the sea state data from Pierson-Moskowitz sea spectrum [12]. 
Table 2. Sea State Parameters[12]

\begin{tabular}{ccccc}
\hline Sea State & $\begin{array}{c}\text { Wind Speed } \\
\text { (knots) }\end{array}$ & $\begin{array}{c}\text { Average wave } \\
\text { period }(\mathrm{sec})\end{array}$ & $\begin{array}{c}\text { Significant wave } \\
\text { height }(\mathrm{m})\end{array}$ & $\begin{array}{c}\text { Average wave } \\
\text { length }(\mathrm{m})\end{array}$ \\
\hline 4 & 18 & 5 & 1.83 & 24.1 \\
5 & 21 & 5.5 & 2.4 & 32 \\
6 & 27 & 7.5 & 4.3 & 56.1 \\
7 & 37 & 10 & 7.62 & 100.13 \\
8 & 49 & 13 & 13.72 & 180.1 \\
\hline
\end{tabular}

On the other hand, due to the reflective nature of sea surface, the reflected component of the signal is influenced by the sea state conditions. It is expected that in calm seaconditions (sea state 1 through 4 ) the signal offset is negligible. However, as the significant wave heights increase the severity of the sea conditions also change and thê signal offset in the receiver is probable to worsen, since the incoming wavefronts will be scattered due to rough sea conditions [13].

\section{Simulation and Results}

In this section, we present the simulation environments and results, with underlying assumptions for the proposed techniques to improve system performance of a ship to ship based maritime communication system. Performance is evaluated, in terms of BER with respect to Eb/No. Maritime wireless communication is different from terrestrial wireless communication, due to sea surface reflection, fading, time delay, Doppler shift, sea state and rocking motion of ships. Our simulation model was applied to that condition under Rician fading channel. For our simution, we use the sea state data from Pierson-Moscowitz sea spectrum. Figure 3 show our simulatiol model. In our simulation model, we assume that the base station is on the berth, relay stations and mobile stations are sailing near the port.

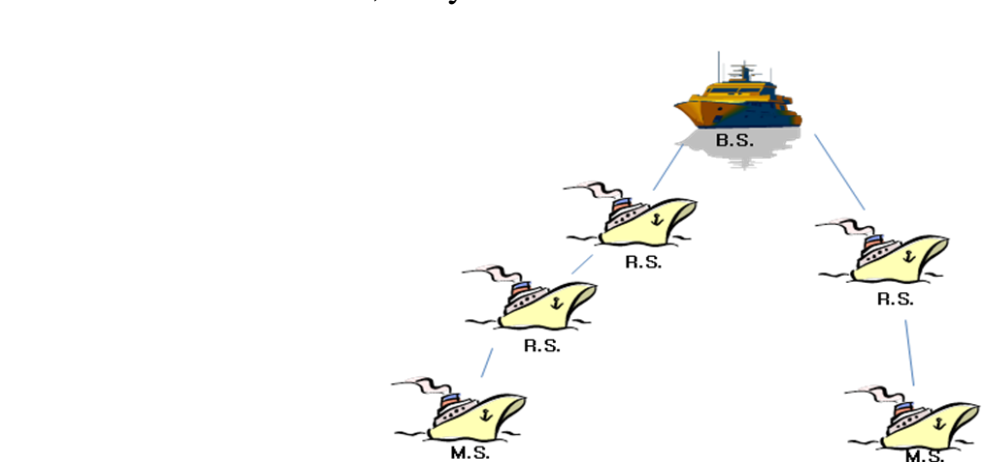

Figure 3. Simulation model

We simulated performance of two transmit, single receive system without power loss, time delay and Doppler shift under sea state conditions at Rician fading channel, with $K=0,5$. Figure 4 and 5 shows simulation results respectively. 


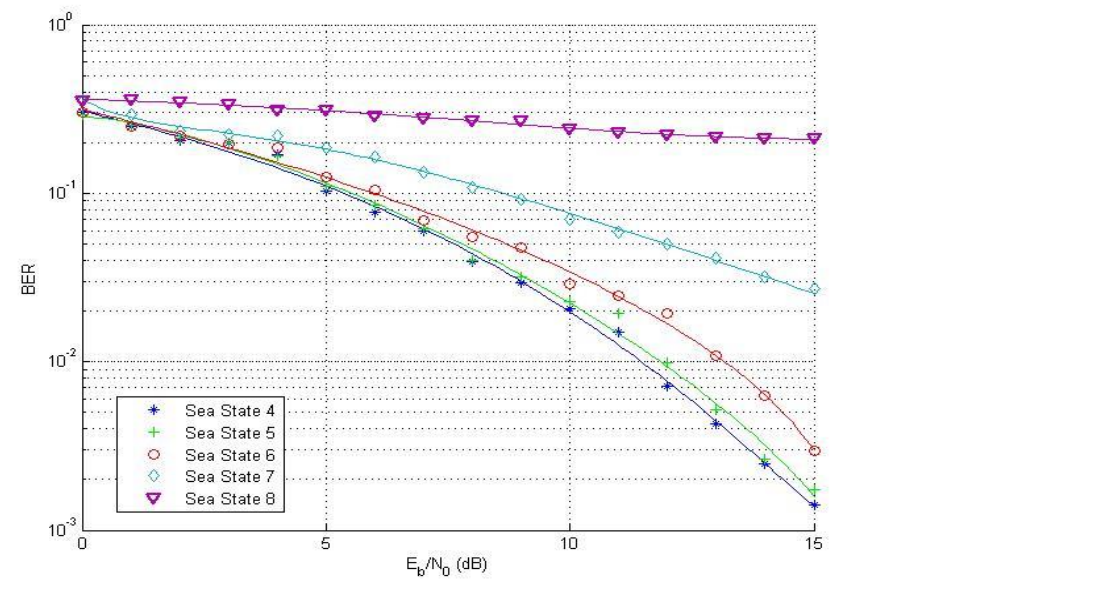

Figure 4. Performance of two transmit, single receive system without power loss, time delay and Doppler shift under sea state conditions, with K=0

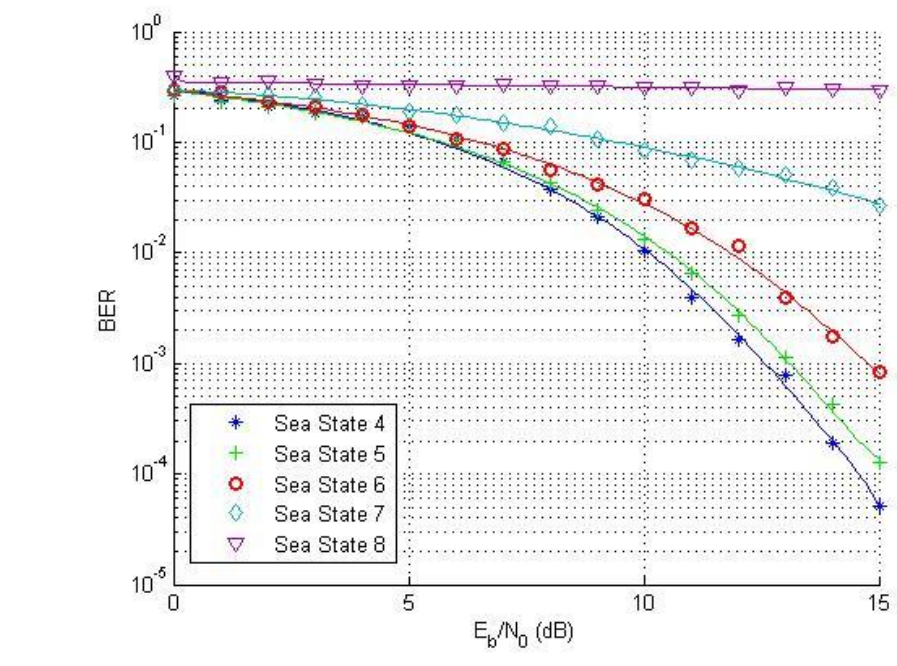

Figure 5. Performance of two transmit, single receive system without power loss, time delay and Doppler shift under sea state conditions, with $K=5$

The figures show the performance of the enhancement achieved with two transmit, single receive systems. It is evident that the performance of the system improves drastically with the diversity. The slope of the BER graph indicates the degree of diversity achieved, this is characterized by the performance improvement in BER. Moreover, the figures above also show the performance of the system with different channel parameters. Hence, it is apparent from the results that sea states 4,5 and 6 are appropriate for communication and performance can be improved in these conditions, however, at sea states 7 and 8 communication becomes unfeasible.

\section{Conclusion}

In this paper, we introduce ship to ship maritime wireless communication based on IEEE $802.16 \mathrm{j}$ for e-Navigation service. Current ship to ship communication systems are based on MF, HF, VHF radios. These maritime radio technologies cannot support the e- 
Navigation service due to lack of bandwidth. To solve this problem, we propose to adopt IEEE 802.16j based maritime ship to ship communication for e-Navigation.

This paper is aimed to improve the performance of IEEE $802.16 \mathrm{j}$ based maritime ship to ship communication for e-Navigation. Performance is evaluated, in terms of BER under various sea states. From the results obtained; it can be concluded that IEEE 802.16j technique is feasible in maritime communication for e-Navigation.

\section{Acknowledgements}

This research was supported by Basic Science Research Program through the National Research Foundation of Korea (NRF) funded by the Ministry of Education (NRF-2009-0093828) and supported by the National Research Foundation of Korea (NRF) funded by the Ministry of Edycation (NRF2011-0023364).

\section{References}

[1] J. G. Andrews, A. Ghosh and R. Muhamed, (Eds.), "Fundamentals of WiMAX. Understanding Broadband Wireless Networking", Prentice Hall, (2007).

[2] L. Nuaymi, (Ed.), "WiMAX: Technology for Broadband Wireless Access, WILEY, (2007).

[3] H. Wang, W. Jia and G. Min, (Eds.), "Effective Cannel Exploitation in IEEE 802.16j Networks for Maritime Communications", Proceedings of the 31st International Conference on Distributed Computing Systems (ICDSC), Minneapolis, MN, (2011), pp. 162-171.

[4] IEEE 802.16j, "IEEE Standard for Local and Metropolitan Area Networks Part 16: Air Interface for Fixed and Mobile Broadband Wireless Access System Multi-hop Relay Specification", IEEE Working Group, (2007).

[5] S. W. Peters and R. W. Heath, “The Future of WiMAX: Multihop Relaying with IEEE 802.16j”, IEEE Communications Magazine, vol. 47, no. 1, (2069), pp. 104-111.

[6] H. Wang and W. Jia, (Eds.) Effective Traffic Control in IEEE 802.16j WiMAX Networks", IEEE 18th International Workshop on Quality of Service (IWQoS 2010), Beijing, PRC, (2010), pp. 1-5.

[7] N. Yun, T. Park, K. Jung, B T. Jang, D. S. Lim and J. M. Kim, "Simulation Analysis on BER Patterns Based Maritime Ad-hoc Networks", Future Information Technology, Application, and Service, Lecture Notes in Electrical Engineering, Vol. 164,(2012), pp. 629-635.

[8] J. S. Pathma untharam, P. Y. Kong, M. T. Zhou, Y. Ge, H. Wang, C. W. Ang, W. Su and H. Harada, (Eds.), "TRITON: High Speed Maritime Mesh Networks", IEEE 19th International Symposium on Personal, Indoor and Mobile Radio Communications, 2008 (PIMRC 2008), (2008).

[9] Wired Ocean-ESA website, http://telecom/esa/int/telecom/www/object/index.cfm?fobjectid=9121.

[10] MIMO, http://en.wikipedia.org/wiki/MIMO.

[11] S. Wen, P. Y. Kong, Shankar J., H. Wang, Y. Ge and C. W. Ang, (Eds.), "A Novel Framework to Simulate Maritime Wireless Communication Networks”, IEEE/MTS OCEANS 2007, Vancouver, BC (2007), pp. 1-6.

[12] Pierson-Moskowitz Sea Spectrum, http://www.syqwestinc.comsupport/Sea\%20State\%20Table.htm.

[13] K. Tushar, S. Yoon and S. R. Lee, "Compensating the Effect of Ship Rocking in Maritime Ship-to-Shore Communication", The Journal of Korea Information and Communication Society, vol. 38, no. 3, (2013), pp. 271-277.

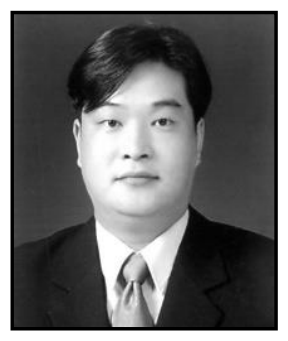

\section{Authors}

\section{Myeong Soo Choi}

$\mathrm{He}$ is a Research Professor at the Institute of Information Science and Engineering Research at the Mokpo National University, Korea. He received his BS, MS, and Ph.D degree in Electronics Engineering at Mokpo National University, Mokpo, Korea, 2000, 2002 and 2009, respectively. His research interests include WPANs, maritime communication, embedded system, ubiquitous sensor network, and wireless communication. 


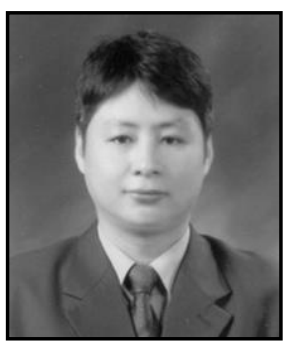

\section{Sun Park}

$\mathrm{He}$ is a research professor at school of Information Communication Engineering at Gwangju Institute of Science and Technology (GIST), South Korea. He received the Ph.D degree in Computer and Information Engineering from Inha University, South Korea, in 2007, the M.S. degree in Information and Communication Engineering from Hannam University, Korea, in 2001, and the B.S. degree in Computer Engineering from Jeonju University, Korea, in 1996. Prior to becoming a researcher at GIST, he has worked as a research professor at Mokpo National University, a postdoctoral at Chonbuk National University, and professor in Dept. of Computer Engineering, Honam University, South Korea. His research interests include Data Mining, Information Retrieval, Information Summarization, Convergence HT and Marine, IoT, and Cloud.

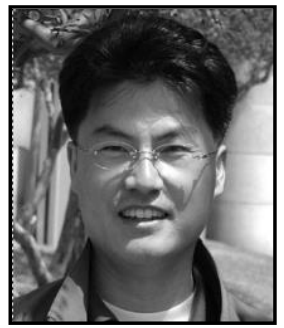

\section{Yeonwoo Lee}

$\mathrm{He}$ is currently a Professor witt the School of Information Engineering at the Mokpo National University, Mokpo, Korea, since September 2005. He has been a Senior Researcher with 4G Mobile Communication team at the Samsung Advanced Institute of Technologies (SAIT), Kiherng, from Januanry 2004 to August 2005.

From October 2000 to December 2003, he has been a Research Fellow with the School of Electronics and Engineering at the University of Edinburgh, UK. From October 2000 to December 2002, he joined core 2 work of Mobile VCE program in UK. He received a MS and Ph.D. in Department of Electronics Engineering from Korea University, Seoul, Korea, in 1994 and 2000, respectively. His research interests are wireless multimedia mobile telecommunication systems, radio resource management, (ad-hoc) multihop relay system, sensor network and particularly their applicable issues to $4 \mathrm{G}$ mobile communication systems and cognitive radio systems.

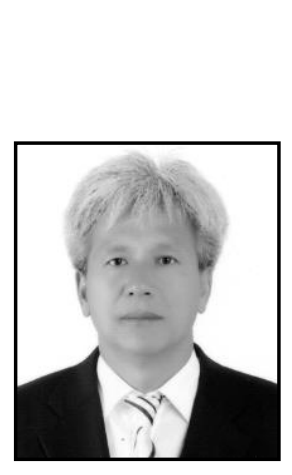

\section{Seong Ro Lee}

He received the B.S. degree in electronics engineering from Korea University, Seoul, Korea, in 1987, and the M.S. and Ph.D. degrees in electrical engineering from Korea Advanced Institute of Science and Technology, Daejeon, Korea, 1990 and 1996, respectively.

In September 1997, he joined the Division of Electronics Engineering, Mokpo National University, Jeonnam, Korea. His research interests include digital communication system, mobile and satellite communications system, applications of telematics, USN and embedded system. He serves as chairman of detection and estimation committee for the Korea Information and Communications Society. 